\title{
EFECTIVIDAD DE LA MENSAJERÍA EXTERNA EN LA ADQUISICIÓN DE LA COMPETENCIA COMUNICATIVA EN UN MODELO DE APRENDIZAJE AUTÓNOMO Y A DISTANCIA EN ESPAÑOL COMO LENGUA EXTRANJERA
}

\author{
EFFECTIVENESS OF EXTERNAL MESSAGING IN THE \\ ACQUISITION OF COMMUNICATIVE COMPETENCE IN A \\ MODEL OF AUTONOMOUS AND DISTANCE LEARNING OF \\ SPANISH AS A FOREIGN LANGUAGE
}

OLGA JUAN LÁZARO

Responsable de Tecnologías Aplicadas y Proyectos Académicos, Instituto Cervantes de Madrid

olgajuan@cervantes.es

\section{RESUMEN}

Los cursos de lenguas a distancia responden adecuadamente a las necesidades de estudiantes que requieren flexibilidad en dedicación horaria. Pero es la modalidad más exigente porque el estudiante se enfrenta a su aprendizaje de manera totalmente autónoma, por lo que sufre una alta tasa de abandono. Estos cursos giran en torno al eje que conforma el material digital y multimedia con el que el alumno interacciona en solitario, y se requiere una conexión de datos y un dispositivo electrónico o móvil. El perfil de alumno demandado implica que éste debe haber desarrollado cierta competencia digital y estrategias de aprendizaje. Una de las claves en este proceso de aprendizaje autónomo es establecer una planificación realista acorde a los plazos de los que dispone el estudiante y al tiempo de dedicación que requiere el curso. En este estudio se exponen los resultados de las acciones llevadas a cabo para mejorar la ratio de estudiantes que finalizan con éxito su curso. El objetivo último sería una propuesta de automatización de los mensajes de planificación, así como diseñar materiales y actividades digitales que hagan hincapié en estrategias de aprendizaje basadas en el objetivo de la planificación como parte del éxito.

Palabras clave: Aprendizaje autónomo, aprendizaje a distancia, competencia digital, aprendizaje a lo largo de toda la vida, aprendizaje de lenguas, estrategias de aprendizaje. 


\begin{abstract}
The online language courses or distance courses respond adequately to the needs of students who require flexibility in time commitment. But it is the most demanding and hard modality because the student learns completely autonomously so it suffers a high rate of abandonment. These courses revolve around the axis that forms the digital and multimedia material with which the student interacts alone, a data connection and an electronic or mobile device. The profile of the student must have developed some digital competence and acquired learning strategies. One of the keys in this process of autonomous learning is to establish a realistic planning according to the terms of available time and dedication required for the course. In this study the results of the actions taken to improve the ratio of students who successfully complete the course are exposed. The ultimate goal would be a proposal of automation planning messages and digital design materials and activities that emphasize learning strategies based on the objective of planning learning process as part of the success.
\end{abstract}

Keywords: Autonomous learning, online learning, digital literacy, life long learning, second languages acquisition, learning strategies.

Recibido: 24.06.2015. Aceptado: 10.08.2015.

\title{
1. INTRODUCCIÓN
}

- $n$ la Sociedad de la Información y la Comunicación los avances tecnológiCos están trayendo consigo una auténtica revolución que afecta a todos los aspectos y facetas de la vida, tanto personal como académica y profesional. En el ámbito de la educación, en el que se encuentra el aprendizaje de lenguas segundas o lenguas extranjeras, las transformaciones vienen desde la aparición de una nueva competencia básica, como es la competencia digital (ISTE, 2007; UNESCO, 2008) hasta la definición de nuevas modalidades de aprendizaje o redefinición de las ya existentes (Juan Lázaro y de Basterrechea, 2004), entre las que se encuentran la enseñanza a distancia con tutor o sin tutor.

La sociedad del siglo XXI demanda una concepción del proceso de aprendizaje que se extiende a lo largo de toda la vida. Para responder a tan ambiciosa meta, se produce una apertura del concepto formativo hacia visiones más globales, en las que la formación no solo es "formal", sino que es también "no formal" e "informal”, y se concibe sin límites temporales, por lo que los dispositivos móviles y la conectividad facilitan la irrupción de nuevas tendencias como el "aprendizaje móvil" a través de tabletas y dispositivos móviles de alta gama. En este escenario, el acceso a contenidos y materiales digitales de calidad es el reto de instituciones educativas y estatales. 
Si nos centramos en una de las modalidades de aprendizaje más flexibles, a distancia y sin tutor o autónomo, uno de los objetivos es conseguir retener al alumno e incrementar el índice de estudiantes que terminan sus cursos (Borges, 2005; Carrizosa, 2014) contribuyendo a la reducción de la tasa de abandono tan alta en estas modalidades. El presente estudio se centra en el impacto que el envío de mensajes de planificación del curso tiene en los alumnos. A la luz del análisis de los datos arrojados, se concretarán propuestas didácticas y automatismos que ayuden al estudiante a ser responsable de su proceso de aprendizaje y a la consecución del objetivo de terminar el curso o los estudios iniciados.

\section{MARCO DE REFERENCIA}

A continuación se presentan los fundamentos teóricos y los conceptos en los que se circunscribe el estudio empírico basado en dos variables principales correlacionadas apriorísticamente.

\subsection{Contexto social tecnológico: aprendizaje a lo largo de toda la vida}

La UNESCO creó en 2006 el "Instituto de la UNESCO para el Aprendizaje a lo Largo de Toda la Vida". Sus proyectos e iniciativas, desde la publicación de los "Informe Faure", 1972, y el "Informe Delors", 1996, han articulado los principios fundamentales del mismo, llegando a considerar que el aprendizaje a lo largo de toda la vida, en cualquiera de sus manifestaciones, es decir, formal, no formal e informal, es clave en el siglo XXI para afrontar los retos del desarrollo social y económico sostenible, además de los problemas mundiales de educación.

En este nuevo contexto social, se anima a las instituciones responsables a investigar y avanzar en modelos que faciliten una formación de calidad en cualquier etapa de la vida. El objetivo último persigue que los ciudadanos estén en disposición de aportar soluciones eficaces a los problemas y situaciones que se dan en la Sociedad de la Información y la Comunicación, caracterizada por una nueva normalidad de profundas y rápidas transformaciones que vienen de la mano de los avances tecnológicos en el acceso a la información y en el desarrollo de las comunicaciones a nivel mundial.

La educación a distancia es una de las opciones que emerge con mayor fuerza para aquellas personas que no pueden llevar a cabo un régimen presencial de formación. En el caso de España, el aprendizaje a lo largo de toda la vida es parte de las "enseñanzas del sistema educativo". En la educación a distancia se distingue una "formación formal" regulada desde el "Centro para la Innovación y Desarrollo de la Educación a Distancia”, CIDEAD, dentro del Ministerio de Educación, 
Cultura y Deporte, y una "educación no formal" promovida desde sistemas organizados desde el propio Ministerio, como "Aula Mentor", en colaboración con Comunidades Autónomas y Ayuntamientos, así como instituciones y organizaciones como el Instituto Cervantes, entre otras.

"Todas las personas deben tener la posibilidad de formarse a lo largo de la vida, dentro y fuera del sistema educativo, con el fin de adquirir, actualizar, completar y ampliar sus capacidades, conocimientos, habilidades, aptitudes y competencias para su desarrollo personal y profesional" "Aprendizaje a lo largo de toda la vida", MECD. Disponible en http://www.mecd.gob.es/educacion-mecd/areas-educacion/sistema-educativo/ ensenanzas/aprendizaje-largo-vida.html).

El presente estudio se enmarca en este contexto: una sociedad tecnológica en que la demanda de adaptación al entorno exige una actitud de formación constante y en que la búsqueda de nuevos modelos pasa por la inclusión total de la modalidad a distancia. En los epígrafes siguientes se concretarán las características de estos enfoques que enmarcan las hipótesis del estudio.

\subsection{Enfoque de enseñanza/aprendizaje de lenguas}

En el ámbito de la enseñanza de lenguas, este trabajo se centrará en los aspectos relacionados con el marco del estudio, a saber, el enfoque de aprendizaje orientado a la acción y, dentro de este, lo relacionado con el aprendizaje estratégico, las implicaciones del concepto de autonomía de aprendizaje y la competencia digital.

\subsubsection{Enfoque orientado a la acción y al aprendizaje estratégico}

Desde la publicación del Marco común europeo de referencia para las lenguas: aprendizaje, enseñanza, evaluación por el Consejo de Europa (MCERL, traducido y adaptado al español por el Instituto Cervantes en el año 2001) hay un consenso generalizado en torno a las directrices que se postulan en el mismo. El enfoque de aprendizaje adoptado se orienta a la acción, en la medida en la que se considera a los alumnos como agentes sociales que aprenden una lengua realizando tareas en las que se ponen en juego todos los recursos cognitivos, emocionales y volitivos que le permiten entender la tarea y conseguir el mejor resultado posible (MCERL, Cap. 2).

El aprendizaje y uso de una lengua comprende el desarrollo de competencias generales y competencias comunicativas. Las competencias generales están formadas por: 
- conocimientos

- destrezas y habilidades (saber hacer)

- competencia existencial (saber ser)

- capacidad de aprender (saber aprender)

Entre los componentes interesa destacar el de "saber aprender", dado que si bien es un componente inherente a todo proceso de aprendizaje, cuando la modalidad elegida es formación a distancia, y, en concreto, sin tutor o sin guía, cobra relativamente mayor importancia, dado que se añade complejidad al proceso y requiere madurez por parte del estudiante (Cabero, 2006; Juan Lázaro, 2001).

Para terminar de conformar una visión completa de los postulados del MCERL, para describir el uso de una lengua, además de las competencias indicadas, hay que sumar las actividades de lengua, los ámbitos de aprendizaje (público, personal, educativo y profesional), tareas, textos y estrategias (estas últimas tanto para la comunicación como para el aprendizaje) que deben formar parte del currículo general.

El interés que se confiere a la parte más estratégica del proceso de aprendizaje y a la capacidad de aprendizaje, se comprende con mayor claridad en el contexto social y tecnológico descrito en el epígrafe 2.1 de este trabajo.

Siguiendo las directrices del MCERL, el Instituto Cervantes ha sido una de las primeras instituciones en desarrollar los inventarios para el español en los diferentes niveles (A1 al C2) en la obra Plan Curricular del Instituto Cervantes (2006), PCIC. El diseño de los materiales para el aprendizaje del español tiene que integrar armónicamente los contenidos descritos en trece inventarios: 1) objetivos generales; 2) gramática; 3) pronunciación; 4) ortografia; 5) funciones; 6) tácticas y estrategias pragmáticas; 7) géneros discursivos y productos textuales; 8) nociones generales; 9) nociones especificas; 10) referentes culturales; 11) saberes y comportamientos socioculturales; 12) habilidades y actitudes interculturales; y 13) procedimientos de aprendizaje. En el inventario decimotercero se presume la inclusión de propuestas didácticas o actividades que ayuden al estudiante a desarrollar estrategias para controlar y regular el proceso de aprendizaje, lo cual le permitirá aplicar estas estrategias a otros momentos del aprendizaje que se promueve a lo largo de toda la vida, más allá del contexto específico en el que se adquieren, por ejemplo, en el caso de este estudio, como parte de las prácticas propuestas en los cursos en línea del Aula Virtual de Español (AVE), tanto en la profundización del conocimiento del español como de otras lenguas, así como en contextos informales y no formales de aprendizaje.

Para este estudio se ha identificado una acción concreta dentro de la "planificación del proceso de aprendizaje”, concibiendo un servicio de comunicación externo que apoye al estudiante en su tarea de planificación y le haga consciente de la carga de trabajo en relación al tiempo que dura el curso. Dentro del inventario de 
"Procedimientos de aprendizaje", se encontraría ubicado dentro del 10 "Relación de procedimientos de aprendizaje", enunciándose como "Planificación del aprendizaje" (PCIC, Instituto Cervantes, 2006, pp. 471).

\subsubsection{Autonomía de aprendizaje}

En la didáctica de lenguas, se define la autonomía como "la capacidad de gestionar el propio aprendizaje" (Holec 1980, citado en el Instituto Cervantes, Diccionario de términos clave de ELE, 2015). Dickinson argumenta a favor del aprendizaje autónomo en el aula o la modalidad presencial con cinco razones:

1. Motivos de tipo práctico (la imposibilidad de asistir regularmente a clase).

2. Las diferencias individuales de los alumnos (relativas no sólo a su actitud, sino también a sus estilos de aprendizaje y a las estrategias que aplican).

3. Los fines educativos (el aprendizaje autónomo promueve el desarrollo de las caracteristicas especificas del buen aprendiente).

4. La motivación (la autonomía estimula al alumno en el sentido de que le otorga libertad).

5. El objetivo de aprender a aprender (la autonomía promueve la reflexión sobre el propio aprendizaje, los procesos y las estrategias utilizadas).

Estas capacidades se adquieren mediante el desarrollo de estrategias metacognitivas que el estudiante debe tener identificadas y que ejercitará en mayor o menor medida, dado que la autonomía en el aprendizaje es intencional, consciente y explícita. En la enseñanza presencial o en el aula con TIC (Tecnologías de la Información y la Comunicación), el rol que se otorga a los materiales digitales y plataformas de aprendizaje o entornos de aprendizaje se halla fuera del aula, dado que el aula es el espacio idóneo para desarrollar la interacción comunicativa de la lengua (Juan Lázaro, 2009a; 2010).

Si se extrapolan los argumentos de Dickinson al aprendizaje a distancia sin un tutor que pueda realizar las funciones de orientador, guía, dinamizador, apoyo al seguimiento (Provencio Garrigós y Juan Lázaro, 2005) se podría decir que el argumento 1), "motivos de tipo práctico", es inherente a esta modalidad de aprendizaje, ya que normalmente el estudiante opta por esta modalidad de aprendizaje sin tutor por necesidad de flexibilidad (Martínez, 2008, 165); respecto al argumento 2), dado que trabaja individualmente, el estudiante lo hará según su estilo de aprendizaje y aplicando estrategias propias; respecto a los argumentos 3), 4) y 5), es decir, desarrollarse como buen aprendiente, no perder la motivación y mejorar la capacidad de aprender a aprender, se puede considerar que serán parte de los retos que garantizarán el éxito del proceso. 
El presente estudio se basa, precisamente, en la identificación de una acción concreta externa al proceso de aprendizaje autónomo en los cursos del AVE, Aula Virtual de Español (ver la descripción de herramientas y servicios diseñados que apoyan el aprendizaje autónomo en Hernández Mercedes, 2010) que se enmarcaría entre estos tres últimos argumentos de Dickinson: una motivación extrínseca que acentúa el peso de la planificación del proceso de aprendizaje.

\subsubsection{Competencia digital}

En las últimas décadas del siglo XX empezó a vislumbrarse la importancia de la competencia digital del ciudadano, pasando a considerarse una de las competencias básicas de cualquier currículo desde el comienzo de la escolarización obligatoria, integrada tanto transversalmente como específicamente en tanto en cuanto una asignatura más. En el transcurso de estos años se han definido los estándares e indicadores a nivel internacional (ISTE 2007; UNESCO 2008, 2013) y si bien no coinciden en la denominación de las subcompetencias que la componen, sí viene a existir consenso en que el ciudadano debe ser hábil en el manejo instrumental de los dispositivos, herramientas y servicios tecnológicos; debe saber integrar su potencial en la resolución de problemas, ayudándose de ellos para buscar información, seleccionarla, ordenarla, clasificarla, transformarla y recuperarla; y deben resultarle útiles para comunicarse y establecer redes de colaboración (Duque de la Torre, A.; Gil Bürman, M.; Juan Lázaro, O. y Sanz de la Morena, C., 2011).

El aprendizaje basado en tecnología es una propuesta en la que el estudiante, inmerso en un entorno tecnológico, ejercita las habilidades, destrezas y se forma en las subcompetencias digitales que le permitirán contribuir a la sociedad mejor preparado. Esta acción formativa se produce, en el estudio realizado, en una plataforma de aprendizaje, el AVE. En ella se alojan los cursos formado por materiales didácticos digitales que combinan multimedia. El estudiante interacciona con dichos materiales digitales aprovechando la riqueza del input multimedia que ofrece y la riqueza de la variedad de la cultura española.

Para terminar, sólo incidir en la importancia de no olvidar este componente en las experiencias de formación en línea, dado que el nivel de competencia informático o instrumental puede frustrar y propiciar el abandono del estudiante en línea (Hara y Kling citado por Borges, 2005).

\subsection{Aprendizaje y TIC (Tecnologías de la Información y la Comunicación)}

Dentro de este apartado, tan en boga ya desde las últimas décadas del siglo pasado, y en el que han nacido disciplinas como CALL (Computer Assisted Language Learning), el presente trabajo se centrará en referenciar el aprendizaje móvil y las 
modalidades de aprendizaje con TIC, en concreto, en la modalidad a distancia (también denominada "en línea”).

\subsubsection{Aprendizaje móvil}

La UNESCO en su reciente publicación Directrices para las politicas de aprendizaje móvil, 2013, propugna dentro de las políticas dirigidas a instituciones y empresas "crear contenidos pedagógicos para utilizarlos en dispositivos móviles y optimizar los ya existentes" como un paso para garantizar una mayor inclusión de las tecnologías en la práctica docente, a la vista de los datos sobre el incremento de uso de dispositivos móviles sobre el de ordenadores (portátiles o de escritorio) entre docentes y estudiantes.

Si el aprendiente dispone de contenidos accesibles desde cualquier lugar y en cualquier momento, se flexibiliza el proceso de aprendizaje en cualquiera de sus modalidades, proporcionando nuevos escenarios fuera de la arquitectura del aula.

Aunque ya nadie se cuestiona la inclusión de las TIC en la educación, la eficacia de la tecnología móvil en los resultados de aprendizaje en la enseñanza presencial presenta los siguientes datos en dos estudios llevados a cabo en Reino Unido y España. En el informe de Attewell, Savill-Smith \& Douch (2009), que recoge los resultados de una experiencia de aprendizaje móvil en el Reino Unido, se concluye que se produce un $8 \%$ de mejora en retención del alumno y un 9,7 en el rendimiento escolar. Los recientes resultados del grupo DIM en la investigación llevada a cabo en España y publicados por P. Marqués (2014), inciden igualmente en los beneficios del aprendizaje móvil: "Según desprende el estudio en más del 90\% de los casos se han logrado mejoras en el aprendizaje y se ha apreciado una mejora en la comprensión de los temas, favoreciendo la creatividad de los alumnos, que trabajan con mayor motivación y de manera más autónoma y alcanzan un alto desarrollo de sus competencias digitales".

Para terminar, no se puede dejar de apuntar cómo en este ecosistema tecnológico emergen nuevas oportunidades y enfoques en la educación, desde la aparición de la conocida como Web 2.0 y las redes sociales (para el AVE ver Juan Lázaro, 2009b), se han sucedido iniciativas en torno a enfoques que recogen este mundo de transformaciones, como, por ejemplo, el denominado "aprendizaje ubicuo" ("u-learning") que se define "como un sistema de aprendizaje en línea (o e-learning) que permite al individuo aprender en cualquier momento y lugar en donde pueda llevar un dispositivo electrónico móvil" (Jones y Jo, 2004, citado por Caldeiro y Schwartzman, 2013). El Instituto de Tecnología de Massachusetts y la Universidad de Harvard lanzaron los cursos MOOC ("Masive Open Online Courses" o CEMA en español, "Cursos en línea masivos y abiertos") que gozan de un gran seguimiento. 
En el compromiso constante por una educación de calidad e innovadora, el Instituto Cervantes se ha sumado a la creación de contenidos digitales accesibles mediante dispositivos móviles, especialmente tabletas. En noviembre de 2014 ha lanzado los cursos de español en línea AVE Global (más información en su portal web http://ave.cervantes.es/AVEGlobal/ave-global.html). La primera modalidad que está a disposición de los usuarios es, precisamente, la de autónomo sin tutor.

\subsubsection{Modalidades de enseñanza: a distancia sin tutor y el aprendizaje autónomo}

La flexibilidad en el diseño de modelos didácticos que incorporan las tecnologías en el aula es enorme (baste pensar en los servicios, materiales, herramientas, etc., que provee Internet, tales como plataformas de aprendizaje, entornos personales de aprendizaje, sin olvidarnos de los recursos multimedia de la web y los servicios de redes sociales en los que día a día se multiplican los post, fotos, vídeos, audios, comentarios...). El esquema básico de modalidades de aprendizaje con tecnologías diferencia tres escenarios: enseñanza presencial o en el aula con TIC, enseñanza semipresencial y enseñanza a distancia (Sánchez Almagro, Dávila Martín, Azorín-Albiñana y Juan Lázaro, 2013; Pellerin y Soler, 2012; García y Ferreira, 2010; Livingstone y Ferreira, 2009; Cabero, 2006; Juan Lázaro y De Basterrechea, 2004; Juan Lázaro, 2001).

El estudio que se está documentando se enmarca en la modalidad más compleja, "a distancia sin tutor", donde el estudiante tiene que haber desarrollado un grado de madurez importante en cuanto a estrategias de aprendizaje y habilidades desarrolladas respecto al aprendizaje autónomo.

El índice de abandono en cursos a distancia es uno de los aspectos que más preocupa a quienes los imparten. Según la periodista Carrizosa (2014), en un artículo en el que presenta un estudio de la Escuela Europea de Dirección de Empresa (EUDE), la deserción se sitúa en torno al 35\% en másteres y posgrados a distancia, y llega hasta el 90\% en los MOOC. Varias son las causas que se apuntan en diferentes estudios (Borges, 2005; Martínez Caro, 2008; Rivera Montalvo, 2011; Carrizosa, 2014), aunque hay que coincidir con ella en que "antes de emprender esta aventura en la Red es preciso un cambio radical de mentalidad en los usuarios porque este tipo de educación ha llegado para quedarse y exige fidelidad, además de desterrar el mito de que estudiar así precisa poco esfuerzo".

El esfuerzo que conlleva la modalidad en línea comienza en la propia planificación del proceso de aprendizaje, el estudiante tiene que autoexigirse una dedicación constante y, se recomienda, concretar unos días y horas determinados, de tal forma que establezca unas rutinas fáciles de identificar y que en la agenda se hayan reservado para el estudio del español. Este es precisamente el aspecto en el que se incide en este estudio. 


\section{DISEÑO Y APLICACIÓN DEL ESTUDIO EMPÍRICO}

En este apartado se detalla el diseño del estudio, se describe la hipótesis que lo ha originado, así como las dos variables principales correlacionadas que se identificaron. A partir de estas variables se definieron las actuaciones que se iban a llevar a cabo así como el análisis de los datos tanto cuantitativos como cualitativos que se han alcanzado, identificándose y modificándose el estudio para adaptarlo y progresar junto a los resultados que se iban obteniendo, lo cual permitió al finalizar el mismo tener una medición más precisa y rica de los resultados respecto a la hipótesis planteada y poder arrojar futuras líneas de investigación en la correlación de mayor número de variables que las inicialmente tenidas en cuenta. Para terminar, se proponen aplicaciones prácticas y futuras líneas de investigación con el objetivo de mejorar los resultados en la modalidad de aprendizaje a distancia autónomo sin tutor.

\subsection{Hipótesis y procedimiento}

El aprendizaje autónomo demanda gran exigencia por parte de los alumnos y es una de las modalidades que arroja mayor índice de abandono. Esta realidad suscitó la siguiente reflexión y su consecuente hipótesis de trabajo: si se apoya el proceso de aprendizaje de los estudiantes ayudándoles a planificar sus horas de estudio, ¿redundará en un mejor índice de finalización del curso? O expresado de otra forma, si se planifica el tiempo de estudio acorde al calendario del curso y, periódicamente, se reajusta la planificación y la carga de trabajo, ¿impactará en un mayor índice de estudiantes que finalizan sus estudios y, por ende, en un menor índice de abandono? La respuesta apriorística a esta hipótesis era afirmativa, este apoyo extrínseco al sistema ayudaría a los estudiantes a tomar conciencia del calendario y de la viabilidad de terminar su curso, aun empezando después, con una dedicación que crecía en intensidad a medida que se acercaba el plazo de finalización del curso.

Para responder a esta hipótesis se diseñaron una serie de actuaciones concretas sobre el denominado "Programa Ciencias sin fronteras" en su primera edición de 2012. Este programa nace del interés del Estado brasileño en becar a sus estudiantes universitarios para realizar un año de sus estudios en universidades españolas. Los estudiantes tenían incluidos en su beca los "Cursos de español general" en línea en la plataforma AVE del Instituto Cervantes previo a su estancia en España, de cara a reforzar su competencia comunicativa en español. El curso de español tenía una duración de tres meses, de agosto a octubre, con una dedicación de 30 horas aproximada por parte del estudiante (depende del ritmo de cada alumno, de su necesidad de repetir cierto tipo de actividades, revisar las fichas gramaticales, etc.). La muestra alcanzó a 1.388 estudiantes brasileños. 
Para llevar a cabo la investigación se diseñaron dos fases diferenciadas para poder medir las variables principales correlacionadas, el impacto de los mensajes externos de planificación del curso y su incidencia en la finalización del curso y, por ello, en la mejora de la adquisición de la competencia comunicativa. La primera fase se centró, por tanto, en el envío de mensajes a los estudiantes. El contenido de estos mensajes era fundamental para el objetivo del estudio, conscientes de ello, se discutió en torno a la concreción de los siguientes parámetros:

- Identificar los momentos apropiados para la remisión de los mensajes.

- Definir el número de mensajes que se iban a enviar.

- Decidir en qué lengua se redactaban los mensajes.

- Concretar los objetivos.

- Establecer cómo se iba a medir el impacto.

Para la segunda fase se consideró de interés completar el estudio con el diseño de una encuesta final en la que se preguntara al estudiante por su valoración de esta acción (el envío de correos sobre planificación). Los resultados cualitativos, en este caso, permitirían completar los cuantitativos y objetivos extraídos de la plataforma.

Además de este interés contrastivo entre las dos fases identificadas, el fin último del estudio es arrojar conclusiones sobre la necesidad o no de implementar este tipo de actuaciones en las modalidades de aprendizaje a distancia sin tutor desarrollando un servicio automático de envío de mensajes sobre planificación en momentos concretos del período de duración del curso, en el caso de la primera fase. En el caso de la encuesta, el objetivo final, dependiendo de la respuesta de los alumnos, se hallaba en recomendaciones sobre la inclusión de actividades o material didáctico específico orientado a las estrategias de buen aprendiente autónomo respecto a la planificación del proceso de aprendizaje.

\subsection{Descripción de las actuaciones}

La primera fase se concretó en una propuesta que consistió en el envío de tres correos con una periodicidad aproximada de tres semanas, en concreto el 22 de agosto, el 10 de septiembre y el 4 de octubre.

A estos correos planificados, y dado el impacto constatado en el transcurso del estudio, se decidió añadir un cuarto correo que fue remitido el 30 de octubre, fecha oficial para la finalización del curso, ampliando en 20 días el plazo de realización del curso y ofreciendo una nueva planificación, esta vez con una dedicación intensiva, que diera una nueva oportunidad a los estudiantes para concluir el curso. 
En el diseño de los correos se propuso que el contenido de los mismos debería atender a los siguientes objetivos:

- Recordar a los estudiantes la planificación inicial del curso (el número de sesiones y temas que tendrían que haber realizado en el momento de recibir el correo).

- Si no habían iniciado el curso, animarles a comenzarlo y facilitarles una nueva planificación conforme al plazo restante.

- Motivarles para continuar con el desarrollo del curso.

- Incidir en el soporte que tenían a disposición con el correo electrónico de atención a los alumnos.

$\mathrm{Al}$ enunciar los objetivos, y en el momento de acometer la redacción de los mensajes, se optó por diferenciar dos modelos de correo, uno dirigido a aquellos alumnos que ya habían iniciado el curso y otro dirigido a aquellos alumnos que no habían entrado todavía al curso. El hecho de mezclar los dos tipos de estudiantes en un único mensaje, simplificaba la actuación pero complicaba en exceso el fin del mensaje. A continuación se ejemplifican los textos de los mensajes.

Ejemplo del correo remitido el 10 de septiembre (segundo correo).

\section{Correo electrónico a los alumnos que no han entrado al curso.}

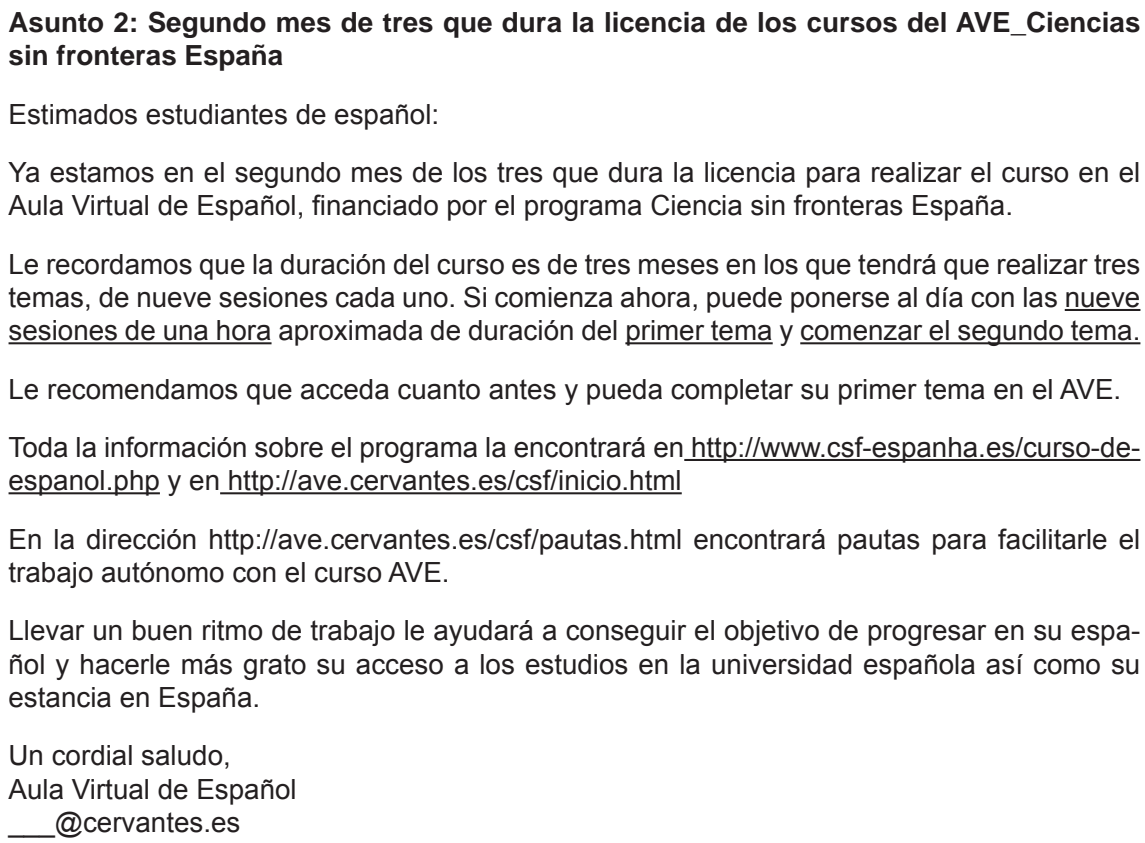

En la dirección http://ave.cervantes.es/csf/pautas.html encontrará pautas para facilitarle el trabajo autónomo con el curso AVE.

Llevar un buen ritmo de trabajo le ayudará a conseguir el objetivo de progresar en su español y hacerle más grato su acceso a los estudios en la universidad española así como su estancia en España.

Un cordial saludo,

Aula Virtual de Español

@cervantes.es 
Efectividad de la mensajería externa en la adquisición de la competencia comunicativa en un modelo de... / O. JUAN LÁZARO

\section{Correo electrónico dirigido a los alumnos que sí han entrado al curso: seguimiento ritmo de trabajo.}

\section{Asunto 2: Segundo mes de tres que dura la licencia de los cursos del AVE_tema 2 en septiembre}

Estimados estudiantes de español:

Ya estamos en el segundo mes de los tres que dura la licencia para realizar el curso en el Aula Virtual de Español, financiado por el programa Ciencia sin fronteras España.

Para llevar un adecuado ritmo de progreso, tendría que haber terminado el primer tema del curso y estar en el segundo tema. Si todavía no lo ha hecho, puede ponerse al día con las nueve sesiones de una hora aproximada de duración del primer tema y comenzar el segundo tema.

Toda la información sobre el programa la encontrará en http://www.csf-espanha.es/curso-deespanol.php y en http://ave.cervantes.es/csf/inicio.html

En la dirección http://ave.cervantes.es/csf/pautas.html encontrará pautas para facilitarle el trabajo autónomo con el curso AVE.

Llevar un buen ritmo de trabajo le ayudará a conseguir el objetivo de progresar en su español y hacerle más grato su acceso a los estudios en la universidad española así como su estancia en España.

Un cordial saludo,

Aula Virtual de Español

@cervantes.es

Los correos fueron siempre remitidos en español (lengua meta objeto de aprendizaje) y portugués (lengua materna de los estudiantes).

Una vez enviados los mensajes, se procedió al análisis del impacto consultando los accesos de los estudiantes en la plataforma (la plataforma AVE cuenta con una herramienta de "Seguimiento y evaluación", en la que se muestra la actividad del estudiante, ver el detalle de su funcionamiento en Olalde Vegas y Juan Lázaro, 2004). En el análisis se contabilizó el número de alumnos que entraban por primera vez a la plataforma tras la recepción del correo en los diez días siguientes. Esta primera hipótesis de estudio fue replanteada a la vista de los resultados en el correo remitido el día 22/08, ya que a partir del quinto día de la recepción del correo el impacto en la actividad del estudiante era prácticamente nulo, es decir, no había nuevos estudiantes que entraran en la plataforma a partir del quinto día. En el reajuste de la medición del impacto se identificó el plazo de una semana para hacer el seguimiento de aquellos estudiantes que reaccionaban entrando a la plataforma por primera vez al recibir el mensaje. Estos resultados vinieron a confirmar la elección respecto a la relación entre las dos variables, como veremos en el siguiente apartado "Resultados" que muestra numéricamente la reacción causa-efecto inmediata. 
La segunda fase del estudio consistió en el diseño de una encuesta en la que se incluyeron cuatro ítems directamente relacionados con el objeto de este estudio y tres ítems preguntando al estudiante si volverían a realizar un curso similar (en este caso, se consideró que la respuesta estaría relacionada con el escenario general concebido y que incluía el envío de los correos sobre la planificación y la propia valoración de la experiencia de aprendizaje autónomo con soporte de un correo de atención al estudiante). La encuesta se completó con ítems sobre la naturaleza de los materiales digitales del curso.

A continuación se ofrece la transcripción de los siete ítems en la encuesta relacionados con el estudio:

\section{Items relacionados con los mensajes de planificación del curso}

\section{Plazo para realizar el curso.}

\begin{tabular}{|l|l|l|l|l|l|l|}
\hline & 1 & 2 & 3 & 4 & 5 & \\
\hline Insuficiente. Falta tiempo. & & & & & & Más que suficiente. Sobraba tiempo. \\
\hline
\end{tabular}

9. Correo de contacto para atender a los alumnos.

O No lo he usado.

O Me han respondido siempre.

O Han tardado en responderme.

O No me han respondido.

10. Correos informativos sobre el ritmo del curso.

O No han sido útiles.

O Han sido algo útiles.

O Han sido útiles. Me han servido para recordar.

O Han sido bastante útiles. Sin ellos no habría continuado.

O Han sido muy útiles. Estoy muy satisfecho.

\section{Items relacionados con la experiencia de realización del curso}

\section{1. ¿Ha terminado el curso?}

O He hecho un $20 \%$ aproximadamente.

O He hecho un $40 \%$ aproximadamente.

O He hecho un $60 \%$ aproximadamente.

O He hecho un $80 \%$ aproximadamente.

O He hecho un $100 \%$ aproximadamente.

\section{Si no lo ha hecho completo, ¿puede explicar el motivo?}


Efectividad de la mensajería externa en la adquisición de la competencia comunicativa en un modelo de... / O. JUAN LÁZARO

\section{3. ¿Nos deja un comentario general sobre su experiencia en este curso?}

\section{4. ¿Volvería a hacer un curso similar?}

O Sí.

O No.

\subsection{Resultados}

Una vez concluido el estudio, los resultados de la primera fase fueron los que se exponen en la Tabla I.

Tabla I. Impacto de los correos de planificación medido dos días y siete días después de su recepción.

\begin{tabular}{|l|l|l|l|}
\hline $\begin{array}{l}\text { Fecha de envío } \\
\text { del correo de } \\
\text { planificación }\end{array}$ & $\begin{array}{l}N^{o} \text { de alumnos } \\
\text { que entran en el } \\
\text { curso DOS DÍAS } \\
\text { después }\end{array}$ & $\begin{array}{l}\text { No de alumnos } \\
\text { que entran en el } \\
\text { curso SIETE DÍAS } \\
\text { después }\end{array}$ & $\begin{array}{l}\text { TOTAL No de } \\
\text { alumnos que entran } \\
\text { en el curso en UNA } \\
\text { SEMANA }\end{array}$ \\
\hline 22 de agosto & 98 estudiantes & 42 estudiantes & 140 estudiantes \\
\hline 10 de septiembre & 19 estudiantes & 11 estudiantes & 30 estudiantes \\
\hline 4 de octubre & 15 estudiantes & 4 estudiantes & 19 estudiantes \\
\hline 30 de octubre & $4 \quad$ estudiantes & 3 estudiantes & 7 estudiantes \\
\hline TOTAL & 136 & 60 & 196 \\
\hline
\end{tabular}

Aunque se consideró insignificante el impacto del envío de correos electrónicos o mensajes a partir del séptimo día después de su recepción para el objeto de estudio (en el caso del correo remitido el 22 de agosto, las mediciones fueron: el quinto día se registró el acceso a los cursos AVE de 21 estudiantes, el sexto día 16 y el séptimo día ya descendió a 5 estudiantes), lo cierto es que en números absolutos el total de alumnos que iniciaron su aprendizaje de español después del primer correo de planificación remitido el 22 de agosto ascendió a 264 (es decir, 
los estudiantes que entraron a la plataforma a partir del 22 de agosto). Esta cifra supone 70 alumnos más que sumar a los 196 que reaccionaron al envío del correo de planificación en el plazo de una semana.

El número de estudiantes que terminó el curso ascendió a 397, por lo que las cifras que estamos manejando respecto al número de estudiantes que reaccionaron a los correos de planificación resultan bastante significativas. A continuación se refleja el tanto por ciento que representa en cada caso el número de alumnos que reaccionó a los correos de planificación comparándolo con el número final de los que terminaron el curso de español:

Tabla II. Datos sobre el porcentaje de alumnos que reaccionó al correo de planificación entrando a la plataforma.

\begin{tabular}{|l|l|l|}
\hline $\begin{array}{l}\text { Fecha de envio } \\
\text { del correo de } \\
\text { planificación }\end{array}$ & $\begin{array}{l}\text { TOTAL No de alumnos que } \\
\text { entran en el curso en UNA } \\
\text { SEMANA. } \\
\text { Total: } 1.388 \text { alumnos }\end{array}$ & $\begin{array}{l}\text { \% alumnos que entran en } \\
\text { el curso UNA SEMANA } \\
\text { después. } \\
\text { \% Sobre el total de alumnos: } \\
1.388\end{array}$ \\
\hline 22 de agosto & 140 estudiantes & $10,08 \%$ \\
\hline 10 de septiembre & 30 estudiantes & $2,16 \%$ \\
\hline 4 de octubre & 19 estudiantes & $1,36 \%$ \\
\hline 30 de octubre & $7 \quad$ estudiantes & $0,50 \%$ \\
\hline TOTAL & 196 & $14,12 \%$ \\
\hline
\end{tabular}

Tabla III. Comparativa datos alumnos.

\begin{tabular}{|l|c|c|}
\cline { 3 - 3 } \multicolumn{2}{l|}{} & $\begin{array}{c}\text { \% Sobre el total de } \\
\text { alumnos: } 1388\end{array}$ \\
\hline $\begin{array}{l}\text { Total de estudiantes que iniciaron su aprendizaje de } \\
\text { español después del primer correo de planificación }\end{array}$ & 264 & $19,02 \%$ \\
\hline Total de estudiantes que terminaron el curso & 397 & $28,6 \%$ \\
\hline
\end{tabular}

Los resultados muestran cómo la respuesta de los estudiantes en la recepción del primer correo de planificación remitido el 22 de agosto ascendió a un 10,08\%. El impacto en el segundo correo no llega a un $50 \%$ respecto al primer correo, situándose tan solo en un 2,16\% la reacción de los alumnos que acceden por 
primera vez al curso casi en el ecuador del mismo, el 10 de septiembre, aproximadamente a las seis semanas.

No obstante, si se considera que sumando el impacto de los correos $\mathrm{N}^{\text {os }} 2,3 \mathrm{y}$ 4 se sitúa en el 4,02\% del total de alumnos, esto significa un 14,105\% del total de alumnos que terminaron el curso. Más significativo es aún el análisis, si se relaciona la cifra total de alumnos que reaccionaron a los siete días con el total de alumnos que terminaron el curso, es decir, el $14,12 \%$ de alumnos que reaccionaron a los correos de planificación una semana después, supone el 49,37\% del total de alumnos que terminaron el curso, es decir, la mitad de los alumnos llevó a buen término sus objetivos de estudio en el curso a distancia gracias a la mediación de los correos de planificación remitidos una vez al mes.

Respecto a la segunda fase, se comentarán los resultados de la encuesta. El 23 de noviembre, una vez terminado el plazo oficial para terminar el curso, incluidos los veinte días de ampliación oficial del mismo, se remitió la encuesta de satisfacción a todos los alumnos que habían iniciado el curso. Se recibieron 247 respuestas.

A continuación se analizará la respuesta a los ítems relevantes para la hipótesis de este estudio y la relación entre ellos.

Sobre el ítem en el que se preguntaba al estudiante sobre la utilidad consciente del envío de correos electrónicos referido al ritmo del curso, se recopilaron un total de 241 respuestas. De las mismas, sólo un 17,01\% reconoce que "Han sido bastante útiles. Estoy muy satisfecho" o "Han sido muy útiles. Estoy muy satisfecho". Un 36,09\% reconocen cierta utilidad: "Han sido útiles. Me han servido para recordar".

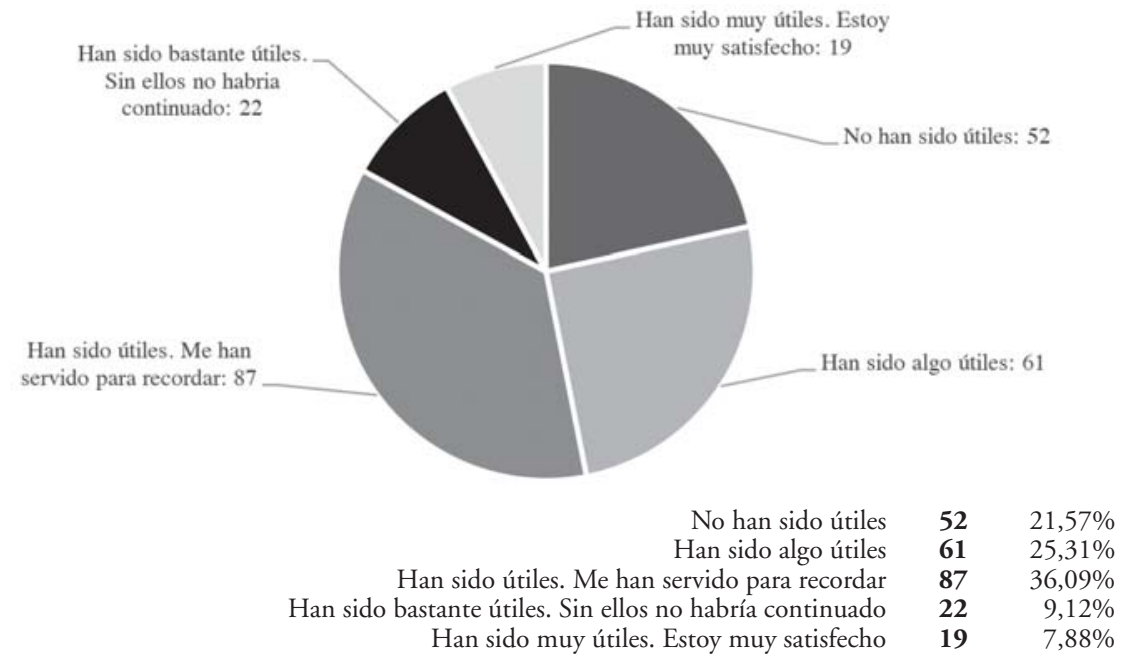

Gráfico1. Encuesta a los estudiantes. Ítem: correos de planificación del curso. 
Cruzando los datos, si se suma el número de respuestas de estas tres categorías $(19+22+87)$ se obtiene un total de 128 , que puede tener relación con los 196 estudiantes que respondieron en el plazo de una semana después de la recepción del correo. En este caso, se propone tener en cuenta la respuesta de aquellos que reconocen que "Han sido algo útiles" sumando 61 nuevos usuarios, lo que hace un total de 189 usuarios para los que el correo de planificación les ha resultado desde algo útil hasta muy útil.

Si establecemos una relación entre los 397 estudiantes que han terminado el curso, el impacto podría llegar al 47,6\%.

En el análisis de estos datos se identificaron otras variables que no habían sido tenidas en cuenta y que se pasarán a concretar para próximas investigaciones. Sería verdaderamente útil contrastar la hipótesis arrojada en la relación de análisis de los datos, es decir, confirmar la relación entre los alumnos que terminan el curso y los que responden al estímulo de los correos de planificación.

En el estudio, llamó la atención el escaso número de correos que se recibieron en la cuenta de atención al usuario. En total ascendieron a 860, lo cual no supone ni un correo por alumno, o, si se relaciona con el número de alumnos que terminaron y se prorratea, la relación sería de dos correos por alumno. Para próximas investigaciones, se recomienda relacionar estos correos con los estudiantes que terminan el curso.

Para terminar, es necesario comentar que a pesar de ser la modalidad más compleja de aprendizaje, y máxime cuando se trata del aprendizaje de segundas lenguas o lenguas extranjeras debido a la necesidad de comunicación en el desarrollo de la competencia de interacción oral o escrita dentro de la competencia comunicativa, un 75,1\% alumnos que respondieron este ítem afirmaron que volverían a hacer un curso similar.

14. ¿Volvería a hacer otro curso similar?

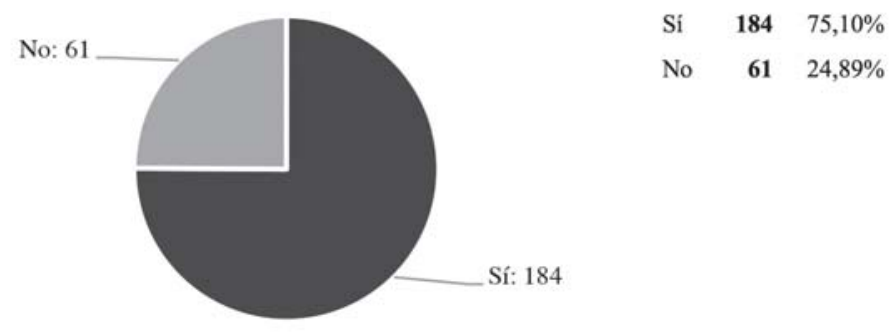

Gráfico 2. Encuesta a los estudiantes. 


\section{CONCLUSIONES}

La modalidad de aprendizaje a distancia y en línea es, sin duda, una de las modalidades que muestra la mayor flexibilidad en el acceso a la formación, encontrándose alineada con las necesidades de una sociedad tecnológica en constante evolución y que demanda el reciclaje continuado de sus ciudadanos en lo que se ha denominado el "aprendizaje a lo largo de toda la vida".

En esta modalidad, normalmente la oferta varía desde una modalidad autónoma de aprendizaje y una modalidad con tutor y/o grupo. La más compleja es la autónoma, aunque obviamente es la más flexible en cuanto a exigencias (no tiene encuentros en síncrono o plazos de finalización y entrega de actividades). La posibilidad de acceder, en este momento, desde dispositivos móviles también flexibiliza el momento de conexión. La complejidad se halla, en este caso, en el perfil del propio estudiante que tiene que ser muy consciente de las exigencias que deben autoimponerse para finalizar el curso, como por ejemplo, es el caso del estudio que nos ocupa, debe realizar una planificación realista del tiempo y prever una dedicación constante al curso.

En este estudio se ha diseñado un escenario para analizar la repercusión que tiene la recepción de correos que recuerdan al estudiante la necesidad de dedicarle tiempo al curso para llevar a buen término sus objetivos, intrínsecamente incide en la motivación en tanto en cuanto planifica el tiempo de acceso que tiene el estudiante, siendo un elemento extrínseco con carga relativa de "acompañamiento" en la experiencia.

El estudio de los datos arroja conclusiones muy halagüeñas respecto a las hipótesis y las variables correlacionadas, ya que sobre el número de alumnos que terminaron el curso, prácticamente la mitad habían respondido positivamente a la recepción de los correos de planificación entrando por primera vez en el curso durante la semana siguiente. Estos resultados animan a proponer dos líneas de actuación. Una primera sería para futuras investigaciones, en las que se podría diseñar en un escenario similar, en una modalidad de aprendizaje autónomo a distancia sin tutor, un estudio que permitiera relacionar individualmente los datos y poder certificar que, efectivamente, el 14,12\% de estudiantes que han reaccionado al envío de correos de planificación en qué tanto por ciento se correlaciona con los que efectivamente han terminado. Y, siendo incluso algo más ambiciosos, qué relación tienen con los datos de la encuesta, es decir, si efectivamente han considerado que era útil o muy útil esta medida arbitrada para el buen logro de sus objetivos (finalizar el curso para mejorar su competencia comunicativa en español).

La otra línea de actuación que propondríamos sería la implementación y automatización de las actuaciones llevadas a cabo en este estudio y explicitadas en este artículo. En este caso concreto irían en dos vertientes, por un lado, un sistema automático que al estudiante le llame la atención sobre la planificación y la dedi- 
cación al curso, y por otro, una serie de actividades, probablemente, para hacer hincapié en la parte estratégica del proceso de aprendizaje.

Consideramos que es una responsabilidad de todos los actantes de los diferentes modelos educativos apoyar el diseño de modalidades que permitan y faciliten el aprendizaje a lo largo de toda la vida, en uno de sus modelos más flexibles, el modelo a distancia sin tutor o curso de aprendizaje autónomo.

\section{REFERENCIAS}

Attewell, J., Savill-Smith, C., \& Douch, R. (2009). The Impact of mobile learning: Examining what it means for teaching and learning, Mobile Learning Network (MoLeNET), LSN. Disponible en http://www.caryloliver.com/Library/ ImpactOfMobileLearning.pdf

AVE Global, (2014). Cursos de español en línea del Instituto Cervantes [en línea]. Instituto Cervantes, Madrid. Disponible en http://ave.cervantes.es/

Borges, Federico (2005). La frustración del estudiante en línea. Causas y acciones preventivas. Digithum [artículo en línea]. UOC. No 7. Disponible en http:// www.uoc.edu/digithum/7/dt/esp/borges.pdf

Cabero, Julio (2006). Bases pedagógicas del e-learning. Revista de Universidad y Sociedad del Conocimiento (RUSC) [artículo en línea]. Vol. 3, $\mathrm{N}^{\circ} 1$. UOC. Disponible en http://rusc.uoc.edu/index.php/rusc/article/viewFile/v3n1-cabero/v3n1-cabero

Caldeiro, G. y Schwartzman, G. (2013). Aprendizaje ubicuo. Entre lo disperso, lo efímero y lo importante: nuevas perspectivas para la educación en línea. Presentado en I Jornadas Nacionales y III Jornadas de Experiencias e Investigación en Educación a Distancia y Tecnología Educativa (PROED). Disponible en http://www.pent.org.ar/institucional/publicaciones/aprendizaje-ubicuo-entrelo-disperso-lo-efimero-lo-importante-nuevas-per.

Carrizosa, Susana (12/01/2014). La deserción puede con los cursos online, El País, Madrid. Disponible en http://economia.elpais.com/economia/2014/01/10/ actualidad/1389360489_728192.html

De Basterrechea. J. P.; Juan Lázaro, O. y Gil Bürmann, M. (2004). El Aula Virtual de Español (AVE): una propuesta de renovación en la enseñanza de E/ LE a través de las TIC. Departamento de Tecnología y Proyectos Lingüísticos. Instituto Cervantes. Congreso Internacional Virtual Educa. Disponible en http://186.113.12.182/catalogo//interna_recurso.php?nt=58615

Diccionario de términos clave de ELE (español lengua extranjera) [en línea] [2015]. Madrid: Instituto Cervantes. Disponible en http://cvc.cervantes.es/ensenanza/ biblioteca_ele/diccio_ele/default.htm

Duque de la Torre, A.; Gil Bürman, M.; Juan Lázaro, O. y Sanz de la Morena, 
C. (2011). La competencia digital docente: ¿qué sabemos y qué podemos hacer?, COMPROFES, Congreso mundial de profesores de español, Instituto Cervantes. Unidad Tecnologías Aplicadas a ELE, Dirección académica. Videotaller y dossier de actividades. Disponible en https://www.academia.edu/1514804/ La_competencia_digital_docente_qu\%C3\%A9_sabemos_y_qu\%C3\%A9_ podemos_hacer y también en https://www.academia.edu/10756024/La_competencia_digital_docente_qu\%C3\%A9_sabemos_y_qu\%C3\%A9_podemos_ hacer_V\%C3\%ADdeotaller

Ferreira, A. F., Salinas, J. G., \& Morales, S. (2014). Using a Task-Based Approach for Supporting a Blended Learning Model for English as a Foreign Language. International Journal of Computer-Assisted Language Learning and Teaching (IJCALLT), 4(1), 44-62. En http://www.irma-international.org/article/usinga-task-based-approach-for-supporting-a-blended-learning-model-for-englishas-a-foreign-language/109830/

García Salinas, J. y Ferreira Cabrera, A. (2010). Entrenamiento en estrategias de aprendizaje de inglés como lengua extranjera en un contexto de aprendizaje combinado. Revista Nebrija de Lingüistica Aplicada, 8(4), 17- 4. Universidad Antonio de Nebrija, Madrid. En http://www.nebrija.com/revista-linguistica/ articulo_2.html

Hernández Mercedes, P. (2010). El Aula Virtual de Español (AVE): un entorno para el aprendizaje autónomo, en Actas de las V Jornadas de estudio sobre materiales didácticos para la enseñanza de idiomas: LA AUTONOMÍA DE APRENDIZAJE. 1 de junio de 2007. Departamento de lenguas y literaturas neolatinas / Departamento de Filología moderna. Universidad de Florencia. Florencia. pp. 75-88. Disponible en http://www.fupress.com/archivio/ pdf/4040.pdf

Instituto Cervantes y Ministerio de Educación, Cultura y Deporte (2001). Marco común europeo de referencia para las lenguas: aprendizaje, enseñanza, evaluación. Anaya: Madrid. Versión electrónica: http://www.cervantes.es/sobre_instituto_cervantes/publicaciones_espanol/espanol_lengua_extranjera/marco_comun_europeo.htm

Instituto Cervantes (2006). Plan Curricular del Instituto Cervantes. Niveles de referencia para el español, Madrid: Biblioteca Nueva. Versión electrónica: http:// cvc.cervantes.es/ensenanza/biblioteca_ele/plan_curricular/default.htm

ISTE (2007). The ISTE NETS and Performance Indicators for Students (NETS•S). Disponible en http://www.iste.org/standards/nets-for-students.aspx. Disponible en español en http://www.eduteka.org/modulos/11/335/59/1

Juan Lázaro, Olga (2001). La red como material didáctico en la clase de ELE. Madrid: Edelsa.

Juan Lázaro, Olga (2009a). La autonomía y el fomento de la responsabilidad del estudiante: Los materiales didácticos digitales e Internet en el aula, en Actas del 
programa de formación para profesorado de ELE 2008. C. Pastor (Coord.). Berlín: Instituto Cervantes. 2009. Próximamente en http://crc.cervantes.es. Disponible en https://www.academia.edu/1081473/La_autonom\%C3\%ADa_y_ el_fomento_de_la_responsabilidad_del_estudiante_los_MDD_Materiales_ Did\%C3\%A1cticos_Digitales_e_Internet_en_el_aula

Juan Lázaro, Olga (2009b). Web 2.0, comunicación y Material Didáctico Digital para el aprendizaje del español: El Aula Virtual de Español el Instituto Cervantes y su actualización, en Revista de Lingüistica Teórica y Aplicada, RLA, Vol. 47 (2)- II Sem. 2009, pp.13-34. Universidad de Concepción, Chile. Disponible en http://www.scielo.cl/pdf/rla/v47n2/ART_02.pdf

Juan Lázaro, O. (2010). Las TIC en el aula de español: la competencia digital y la autonomía del estudiante, Mosaico, Revista para la promoción y Apoyo a la Enseñanza del Español, Núm. 25-junio 2010. Consejería de Educación en Bélgica, Países Bajos y Luxemburgo, Ministerio de Educación de España, pp. 4-11. En https://sede.educacion.gob.es/publiventa/detalle.action? $\operatorname{cod}=13645$ y en https://www.academia.edu/1273908/_Las_TIC_en_el_aula_de_ espa\%C3\%B1ol_la_competencia_digital_y_la_autonom\%C3\%ADa_del_estudiante_Revista_Mosaico_n.o_25_pp._4-11

Juan Lázaro, O., y De Basterrechea. J. P. (2004). El aula, la enseñanza semipresencial y a distancia: El Aula Virtual de Español en Internet en Pastor, C. (Coord.): Actas del programa de formación para profesorado de ELE 2003-2004. Múnich: Instituto Cervantes Múnich, pp. 105-122. Disponible en http:// cvc.cervantes.es/ensenanza/biblioteca_ele/publicaciones_centros/PDF/munich_2003-2004/09_juan.pdf

Livingstone, K.A. y Ferreira, A. (2009). La efectividad de un modelo metodológico mixto para la enseñanza-aprendizaje de español como lengua extranjera. Boletín de Filología, v. 44, n. 2 (2009): 89-118. Disponible en http://www. al-dia.cl/sistema/tablas/listar.asp? $\mathrm{r}=2581 \#$

Marqués, P. (2014). Uso educativo de las tabletas digitales. Metainvestigación 20132014. Grupo DIM, Universitát Jaume I, Castellón. Disponible en http:// es.slideshare.net/peremarques/tabletas-digitales-uso-educativo-metainvestigacin-dim y http://peremarques.net/tabletasportada.htm

Martínez Caro, Eva (2008). E-learning: un análisis desde el punto de vista del alumno, RIED, v.II:2, pp.151-168. [Artículo en línea]. https://scholar.google. es/scholar?cluster $=4754977001481672220 \& \mathrm{khl}=\mathrm{es} \&$ as_sdt $=0,5 \& \mathrm{sciodt}=0,5$

Ministerio de Educación, Cultura y Deporte: Aprendizaje a lo largo de la vida [contenido en línea]. [Consulta: 15/01/2015]. En http://www.mecd.gob.es/ educacion-mecd/areas-educacion/sistema-educativo/ensenanzas/aprendizajelargo-vida.html

Olalde Vegas, S.Ma y Juan Lázaro, O. (2004). Distance-learning Spanish courses: a follow-up and assessment system, Studies in Language Testing 18, European 
Efectividad de la mensajería externa en la adquisición de la competencia comunicativa en un modelo de... / O. JUAN LÁZARO

Language Testing in a Global Context, Cambridge University Press, pp. 221236.

Pellerin, M. \& Soler, C. (2012). Using the Spanish Online Resource Aula Virtual de Español (AVE) to Promote a Blended Teaching Approach in High School Spanish Language Classrooms, DJLT \& RCAT (Canadian Journal of Learning and Technology / La revue canadienne de l'apprentissage et de la technologie), Vol. 38 (1). Disponible en http://www.cjlt.ca/index.php/cjlt/article/view/597

Provencio Garrigós, Herminia y Juan Lázaro, Olga (2005). Funciones del tutor y compromisos del alumno en la docencia semipresencial y a distancia de español (E/LE), Frecuencia-L, Madrid: Editorial Edinumen. En http://issuu.com/ hprovencio/docs/h-provencio_0-juan-lazaro

Rivera Montalvo, D. (2011). Factores que Inciden en la Retención o Deserción del Estudiante a Distancia. Nova Southeastern University. http://ponce.inter. edu/cai/tesis/derivera/index.pdf

Sánchez Almagro, Ma L.; Dávila Martín, C.; Azorín-Albiñana, A. y Juan Lázaro, O. (2013). El Aula Virtual de Español como recurso del aprendizaje semipresencial en el Centro de Personas Adultas, V Jornadas iTIC 2013, Madrid. Disponible en http://www.iticlab.es/camarote/?page_id=361

UNESCO, Organización de las Naciones Unidas para la Educación, la Ciencia y la Cultura (2008). Estándares de competencias en TIC para docentes. Disponible en http://portal.unesco.org y http://cst.unesco-ci.org/

UNESCO (2013). Directrices para las políticas de aprendizaje móvil. Disponible en http://unesdoc.unesco.org/images/0021/002196/219662S.pdf

UNESCO. Políticas y Estrategias de Aprendizaje a lo Largo de Toda la Vida [contenido en línea]. [Consulta: 15/01/2015]. En http://uil.unesco.org/es/portal/ areas-de-trabajo/politicas-y-estrategias-de-aprendizaje-a-lo-largo-de-toda-lavida/ 